\title{
Association Between Multiple Lines of Active Therapy and Prognosis in Esophageal Squamous Cell Carcinoma
}

This article was published in the following Dove Press journal: Cancer Management and Research

\author{
Ying Yang \\ Jun Jia \\ Zhiwei Sun \\ Chuanling Liu \\ Yanjie Xiao \\ Jing Yu \\ Feng Du (iD) \\ Xiaodong Zhang
}

Key Laboratory of Carcinogenesis and Translational Research (Ministry of Education, China), The VIP-II

Gastrointestinal Cancer Division of Medical Department, Peking University Cancer Hospital \& Institute, Beijing, China
Correspondence: Xiaodong Zhang Key Laboratory of Carcinogenesis and Translational Research (Ministry of Education, China), The VIP-II Gastrointestinal Cancer Division of Medical Department, Peking University Cancer Hospital \& Institute, Beijing, China Email zhangxd0829@I63.com
Purpose: Esophageal squamous cell carcinoma (ESCC) is a critical threat to health and life. More than half of ESCC patients have recurrent or metastatic disease. Most late-stage patients undergo first-line treatment but experience further progression. Many of these patients have good performance status and are able to receive second-line therapy and even further treatments rather than best supportive care. Our analysis aimed to explore whether multiple lines of active treatment are beneficial in ESCC patients.

Methods: We conducted a retrospective cohort study. Univariate and multivariate survival analyses were used to identify whether the number of active treatment lines was related to prognosis. All analyses and the corresponding survival curves were based on the Cox proportional hazard regression model and the Kaplan-Meier method. Comparisons between groups were conducted using the $t$-test, chi-square test, and Fisher's exact test, as applicable. Results: Of a total of 138 patients with recurrent or metastatic disease, 66 (61.1\%) received one line of active treatment, and 42 (38.9\%) patients received two and more lines. Multiple lines of active therapy were statistically significantly associated with better prognosis (crude hazard ratio (HR) $(95 \%$ confidence interval $(\mathrm{CI}))=0.21(0.06-0.73))$, even after adjusting for relevant confounders (adjusted HR $(95 \% \mathrm{CI})=0.19(0.04-0.86))$. More grade 3-4 hepatotoxicity patients were observed in the multiple-line treatment group $(\mathrm{p}=0.033)$. A small number of patients were cured by palliative management; these patients were more likely to have received both systematic and local treatment than other patients with repeated progression $(9 / 15$ versus $40 / 117, \mathrm{p}=0.051)$.

Conclusion: Multiple lines of active treatment are related to prolonged survival in recurrent and metastatic ESCC patients, and adverse effects are acceptable. Comprehensive therapy modalities are recommended.

Keywords: multiple lines of active treatment, esophageal squamous cell carcinoma, prognosis

\section{Introduction}

Esophageal cancer is a serious threat to health and life. Globally, there were an estimated 572,034 new cases in 2018, making it the seventh most common malignant tumor, and 508,585 deaths, making it the sixth leading cause of cancer deaths. ${ }^{1}$ The most recently published Chinese statistics showed that there were 477,900 new cases of esophageal cancer in 2015, and 375,000 deaths; thus, it was the third most common cancer and the fourth leading cause of cancer deaths in China. ${ }^{2}$

In contrast to Western countries, where most esophageal cancers are adenocarcinomas, the major pathology type of esophageal cancer in China is squamous cell 
carcinoma. ${ }^{3}$ Esophageal squamous cell carcinoma (ESCC) patients are recommended to treat with comprehensive treatment modalities, including but not limited to surgery, radiotherapy, and systematic treatment, and even interventional therapy and radiofrequency ablation, aiming to improve prognosis and prolong survival. Despite advances in surgical and radiotherapy techniques, and the integrated application of various treatment means, the 5-year survival rate of ESCC patients remains less than $20 \%{ }^{2}$ Of the early- and middlestage ESCC patients who are able to receive radical treatment such as esophagectomy or definite (chemo)radiotherapy, around 28-74\% eventually experience relapse. ${ }^{4}$ Around $40 \%$ of all patients at initial diagnosis have distant metastatic disease, which is considered to be incurable. ${ }^{5,6}$ For late-stage patients, the natural course is $6-8$ months, and those who undergo first-line chemotherapy with platinum plus paclitaxel or fluorouracil have overall survival (OS) of less than 1 year. The 5-year survival rate of patients with recurrent or metastatic disease is only $5-7 \%{ }^{7,8}$

In recurrent or late-stage patients, after the first-line palliative management, most experience inevitable progression; however, there is a lack of solid evidence supporting subsequent aggressive therapies, and the choice of treatment modality is institution-specific; therefore, the benefits of second- and later-line active treatments remain uncertain and worthy of further exploration. ${ }^{9}$

Our study was the first to systematically explore the association between multiple lines of active therapy and prognosis in ESCC patients. ${ }^{10-14}$

\section{Methods}

\section{Population}

From August 2012 to February 2016, a total of 193 esophageal cancer patients were enrolled in the VIP-II Gastrointestinal Cancer Division of Medical Department, Peking University Cancer Hospital \& Institute. There were 169 ESCC cases, 10 neuroendocrine carcinomas, and 3 adenocarcinomas, as well as 8 mixed-pathology carcinomas and 3 synchronous primary cancers. All cases were historically confirmed. Of the 169 ESCC patients, 119 received radial therapies, to be specified, 55 underwent surgery, 61 received definite chemoradiotherapy, and 3 received definite radiotherapy. The other 50 patients did not receive radical treatment owing to distant metastasis, contraindication, or patients' choice. Twenty-eight patients did not experience relapse after radical treatment, and three were lost to followup. Further analyses were conducted in the 138 ESCC patients with relapse or metastasis (Figure 1).

\section{Study Design}

This was a retrospective cohort study. The outcome was OS, that is, days from the date of confirmed relapse or metastasis to the date of death or the date of the last follow-up on August 2nd, 2018. The major research interest was the effect of cumulative active treatment lines. Following each progression, each aggressive treatment modality (e.g., chemotherapy, radiotherapy, or interventional therapy) but not best supportive care (BSC) was counted as one line of active

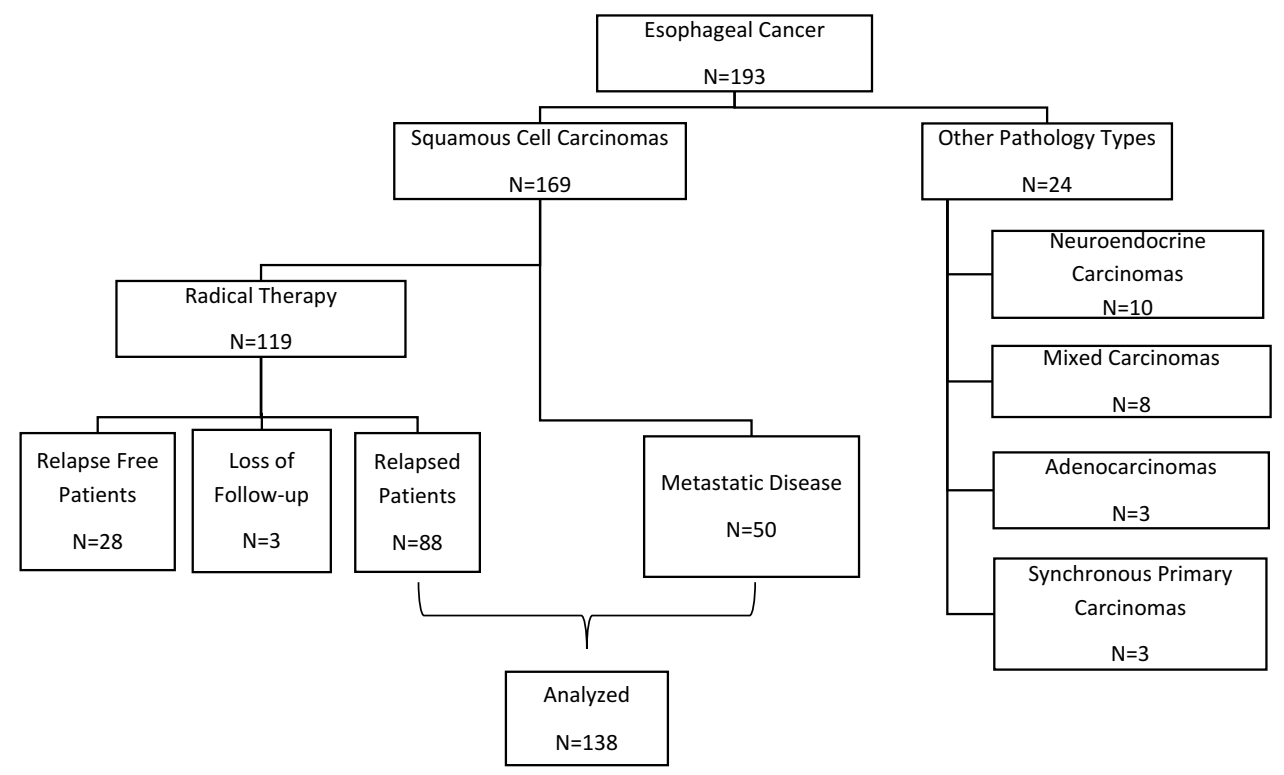

Figure I Flowchart of patient selection. 
treatment, and cumulative treatment lines was calculated as the sum of all active lines per patient. Other relevant variables were defined as follows. Based on examination by endoscopy, cervical ESCC was located from $15 \mathrm{~cm}$ to less than $20 \mathrm{~cm}$, upper thoracic ESCC from $20 \mathrm{~cm}$ to less than $25 \mathrm{~cm}$, middle thoracic ESCC from $25 \mathrm{~cm}$ to less than $30 \mathrm{~cm}$, and lower thoracic ESCC located at $30 \mathrm{~cm}$ to less than $40 \mathrm{~cm}$ from the incisor to the center of the tumor mass. "Multiple lesions" were defined as the presence of several malignant lesions separated by tissues that were normal on gross examination. "Lymphatic and hematogenous metastases" were assessed by a variety of tools, including esophageal ultrasonography, computed tomography, and magnetic resonance imaging or positron emission tomography-computed tomography. According to American Joint Committee on Cancer staging manual 7 th edition, ${ }^{15}$ based on either postoperative pathology reports or clinical assessments at diagnosis, stage was stratified into three categories - stage I and II as "early stage", stage III and local advanced disease as "advanced stage", and stage IV as "hematogenous metastasis". Furthermore, in this study, we used first progression-free survival (PFS) to denote the time interval from the date of relapse to the date of the first progression in radical treatment patients, or from the date of diagnosis to the date of the first progression in non-radical treatment patients.

\section{Statistical Analysis}

Comparisons between two groups used $t$-test, Chi-square test, or Fisher's exact test, as applicable. To identify whether active treatment lines were related to prognosis, univariate and multivariate survival analyses, adjusting for confounders, were performed by Cox proportional hazard regression. All factors included in the regression model were tested for proportional hazard assumptions, and no violations were detected (Supplementary Table 1). The corresponding survival curves were plotted by the Kaplan-Meier method. Estimated follow-up period was calculated with reverse Kaplan-Meier method. All statistical analyses were two-sided, and a p-value less than 0.05 was considered to indicate statistical significance. Results were presented in terms of crude and adjusted hazard ratios (cHRs and aHRs) and 95\% confidence intervals (95\% CIs). All analyses and plots used SAS 9.4 (SAS Institute Inc., Cary, NC, USA).

All patients in our study provided written informed consent to review their medical records for scientific research purpose; and the study was approved by the
Institutional Review Board of Peking University Cancer Hospital \& Institute.

\section{Results}

A total of 138 ESCC patients were included. The estimated median follow-up was 1352 (927-1709) days. These comprised with 88 relapsed patients, including 47 (34.1\%) patients receiving surgery, 38 (27.5\%) receiving definite chemoradiotherapy, and $3(2.2 \%)$ receiving definite radiotherapy, as well as $50(36.2 \%)$ metastasis patients who did not undergo radical treatment.

The median age at diagnosis was 59.5 years, ranging from 43 to 80 years. There were $112(81.2 \%)$ male and 26 (18.8\%) female patients. Thirty-one $(23.5 \%)$ patients had early-stage disease; the majority $(73,55.3 \%)$ were advanced stage, and 28 (21.2\%) had hematogenous metastasis. Fourteen (10.4\%) patients had multiple esophageal lesions; the other $121(89.6 \%)$ had single lesion, comprising $7(5.8 \%)$ cervical esophageal carcinomas, $8(6.6 \%)$ upper thoracic esophageal carcinomas, 31 (25.6\%) middle thoracic esophageal cancers, and 75 (62.0\%) lower thoracic esophageal cancers.

In regard to palliative management modalities, of the 88 patients who received radical treatment and relapsed, $29(33.3 \%)$ underwent both systematic and local treatment, such as radiotherapy, which was the most common local treatment, radiofrequency ablation, and interventional therapy. Twenty-one $(24.1 \%)$ patients received systematic treatment only, and $8(9.2 \%)$ received local therapy only; the remaining 29 (33.3\%) patients chose BSC as the first treatment modality after relapse. Of the 50 patients with metastasis, $20(40.0 \%)$ received both systematic and local treatment, $26(52.0 \%)$ received systematic treatment only, $3(6.0 \%)$ received local treatment only, and $1(2.0 \%)$ chose BSC.

Taking all relapsed and metastasis patients together, after the first therapy, 15 (11.4\%) patients did not progress again; and 117 patients experienced a new progression, most of whom (71.8\%) still had Eastern Cooperative Oncology Group (ECOG) status of 0-2. However, only 42 patients received more aggressive treatment, that is, systematic treatment combined with local therapy in 7 $(5.3 \%)$ cases, systematic treatment only in $26(19.7 \%)$ cases, and local treatment in $9(6.8 \%)$ cases; more than half of the patients $(56.8 \%)$ chose BSC.

Patients who progressed more than once were less likely to receive active treatment other than $\mathrm{BSC}$, and most underwent systematic treatment only. For example, 
113 relapsed and metastasis patients had clear records of a second progression, and among them, only 15 patients received aggressive therapy, of whom 12 chose systematic treatment only. Of the 110 patients who experienced a third deterioration, only four $(3.0 \%)$ could receive systematic treatment; all the others chose BSC only.

Excluding patients lost to follow-up and those choosing only BSC all the time, $66(61.1 \%)$ patients received one line of active treatment, $28(25.9 \%)$ received two, 10 $(9.3 \%)$ received three, and $4(3.7 \%)$ patients received four lines of active treatment.

The mean first PFS was 7.9 months, with a median value of 7.0 months and a range from 1.0 to 31.0 months. At the last follow-up, $109(79.0 \%)$ patients had died, 22 $(15.9 \%)$ were still alive, and $7(5.1 \%)$ had been lost to follow-up. The median survival was 345.5 days (range 27-2256 days). All these results are shown in Table 1.

Several clinicopathological features were analyzed to determine their relationship with prognosis. Esophageal lesion numbers and location were borderline significantly associated with survival. Multiple lesions were associated with shorter survival $(\mathrm{cHR}(95 \% \mathrm{CI})=3.37(0.90,12.57)$, $\mathrm{p}=0.071)$; and upper thoracic ESCC was related to worse prognosis $(\mathrm{cHR}(95 \% \mathrm{CI})=3.36(0.83,13.65), \mathrm{p}=0.091)$ compared with lower thoracic ESCC.

Regarding the lines of active treatments, two and more lines of aggressive therapy were significantly related to longer survival $(\mathrm{cHR} \quad(95 \% \quad \mathrm{CI})=0.21 \quad(0.06,0.73)$, $\mathrm{p}=0.014)$ compared with one-line active treatment. Furthermore, adjusting for age, gender, stage, and previous radical treatment, multiple lesions and tumor location, multiple lines of therapy were still statistically significantly associated with better survival, that is, the adjusted HR and $95 \%$ CI of patients receiving two and more lines of active therapy were 0.19 and $0.04-0.86$, as shown in Table 2. The corresponding survival curves are shown in Figure 2.

We also analyzed whether two and more lines of aggressive therapy were related to more severe adverse effects. The results are shown in Table 3. Concerning grade 3-4 side effects, hepatotoxicity was observed in 4 out of 15 patients in the multiple-line treatment group, versus none of the 18 patients receiving one-line treatment only $(p=0.033)$. No treatment-related deaths were observed in either group.

Finally, we examined the characteristics of the 15 recurrent and metastasis patients who were cured by first-line therapy and had long-term survival. Except for the treatment
Table I Characteristics of Relapsed or Metastasis Patients $(\mathrm{N}=138)$

\begin{tabular}{|c|c|c|}
\hline & $\mathbf{N}$ & $\%$ \\
\hline Age (Years) & & \\
\hline Mean & $60.0 \pm 8.3$ & \\
\hline Median & 59.5 & \\
\hline Range & $43-80$ & \\
\hline Gender & & \\
\hline Male & 112 & 81.2 \\
\hline Female & 26 & 18.8 \\
\hline Stage & & \\
\hline Early & 31 & 23.5 \\
\hline Advanced & 73 & 55.3 \\
\hline Hematogenous metastasis & 28 & 21.2 \\
\hline Missing & 6 & \\
\hline Multiple Lesions & & \\
\hline Yes & 14 & 10.4 \\
\hline No & 121 & 89.6 \\
\hline Missing & 3 & \\
\hline Location (Single Lesion Only) & & \\
\hline Cervical & 7 & 5.8 \\
\hline Upper thoracic & 8 & 6.6 \\
\hline Middle thoracic & 31 & 25.6 \\
\hline Lower thoracic & 75 & 62.0 \\
\hline Radical Treatment & & \\
\hline Surgery & 47 & 34.1 \\
\hline Definite chemoradiation & 38 & 27.5 \\
\hline Definite radiation & 3 & 2.2 \\
\hline No radical treatment & 50 & 36.2 \\
\hline $\begin{array}{l}\text { First Treatment Modality in Relapsed Patients } \\
(\mathrm{N}=88)\end{array}$ & & \\
\hline Systematic and local treatment & 29 & 33.3 \\
\hline Systematic treatment & 21 & 24.1 \\
\hline Local treatment & 8 & 9.2 \\
\hline Supportive care & 29 & 33.3 \\
\hline Missing & I & \\
\hline First Treatment Modality in Metastasis & & \\
\hline Patients $(\mathrm{N}=50)$ & & \\
\hline Systematic and local treatment & 20 & 40.0 \\
\hline Systematic treatment & 26 & 52.0 \\
\hline Local treatment & 3 & 6.0 \\
\hline Supportive care & I & 2.0 \\
\hline ECOG After First Progression & & \\
\hline 0 & 0 & 0 \\
\hline 1 & 44 & 37.6 \\
\hline 2 & 40 & 34.2 \\
\hline 3 & 27 & 23.1 \\
\hline 4 & 6 & 5.1 \\
\hline Missing & 21 & \\
\hline
\end{tabular}

(Continued) 
Table I (Continued).

\begin{tabular}{|c|c|c|}
\hline & $\mathbf{N}$ & $\%$ \\
\hline \multicolumn{3}{|c|}{ Second Treatment Modality (Relapse and } \\
\hline \multicolumn{3}{|c|}{ Metastasis Together) } \\
\hline Systematic and local treatment & 7 & 5.3 \\
\hline Systematic treatment & 26 & 19.7 \\
\hline Local treatment & 9 & 6.8 \\
\hline Supportive care & 75 & 56.8 \\
\hline No progression & 15 & 11.4 \\
\hline Missing & 6 & \\
\hline \multicolumn{3}{|c|}{ Third Treatment Modality (Relapse and } \\
\hline \multicolumn{3}{|l|}{ Metastasis Together) } \\
\hline Systematic and local treatment & I & 0.8 \\
\hline Systematic treatment & 12 & 9.1 \\
\hline Local treatment & 2 & 1.5 \\
\hline Supportive care & 98 & 74.2 \\
\hline No progression & 19 & 14.4 \\
\hline Missing & 6 & \\
\hline \multicolumn{3}{|c|}{ Fourth Treatment Modality (Relapse and } \\
\hline \multicolumn{3}{|l|}{ Metastasis Together) } \\
\hline Systematic and local treatment & 0 & 0.0 \\
\hline Systematic treatment & 4 & 3.0 \\
\hline Local treatment & 0 & 0.0 \\
\hline Supportive care & 106 & 80.3 \\
\hline No progression & 22 & 16.7 \\
\hline Missing & 6 & \\
\hline \multicolumn{3}{|l|}{ Active Therapy Line Counts } \\
\hline Once & 66 & 61.1 \\
\hline Twice & 28 & 25.9 \\
\hline Three times & 10 & 9.3 \\
\hline Four times & 4 & 3.7 \\
\hline BSC only and missing & 30 & \\
\hline \multicolumn{3}{|l|}{ First PFS (Months) } \\
\hline Mean & $7.9 \pm 5.7$ & \\
\hline Median & 7.0 & \\
\hline Range & $1.0-31.0$ & \\
\hline \multicolumn{3}{|l|}{ Follow-Up } \\
\hline Death & 109 & 79.0 \\
\hline Survival & 22 & 15.9 \\
\hline Loss to follow-up & 7 & 5.1 \\
\hline \multicolumn{3}{|l|}{ Survival (Days) } \\
\hline Mean & $463.0 \pm 418.7$ & \\
\hline Median & 345.5 & \\
\hline Range & $27-2256$ & \\
\hline
\end{tabular}

modality, we did not find statistically significant differences to distinguish these patients. Notably, survivors were more likely to have received both systematic and local treatment $(9 / 15(60 \%)$ versus $40 / 117(34 \%), p=0.051)$.
Table 2 Associations Between Selected Clinicopathological Factors and Survival

\begin{tabular}{|c|c|c|}
\hline & HR (95\% Cl) & p-value \\
\hline Age & $1.04(0.98,1.09)$ & 0.177 \\
\hline \multicolumn{3}{|l|}{ Gender } \\
\hline Male & 1.00 & \\
\hline Female & $1.29(0.49,3.37)$ & 0.610 \\
\hline \multicolumn{3}{|l|}{ Stage } \\
\hline Early & 1.00 & \\
\hline Advanced & I.3I $(0.45,3.85)$ & 0.625 \\
\hline Late & $0.68(0.16,2.95)$ & 0.611 \\
\hline \multicolumn{3}{|l|}{ Multiple Lesions } \\
\hline No & 1.00 & \\
\hline Yes & $3.37(0.90,12.57)$ & 0.071 \\
\hline \multicolumn{3}{|l|}{ Location } \\
\hline Lower thoracic & 1.00 & \\
\hline Middle thoracic & $2.17(0.52,9.03)$ & 0.288 \\
\hline Upper thoracic & $3.36(0.83,13.65)$ & 0.091 \\
\hline Cervical & $2.15(0.44,10.40)$ & 0.343 \\
\hline \multicolumn{3}{|l|}{ Radical Treatment } \\
\hline No & 1.00 & \\
\hline Yes & $1.09(0.43,2.78)$ & 0.854 \\
\hline First PFS & $0.91(0.79,1.05)$ & 0.187 \\
\hline \multicolumn{3}{|l|}{ Active Treatment Lines } \\
\hline One & 1.00 & \\
\hline Two and more & $0.21(0.06,0.73)$ & 0.014 \\
\hline \multicolumn{3}{|l|}{ Adjusted Active Treatment Lines* } \\
\hline One & 1.00 & \\
\hline Two and more & $0.19(0.04,0.86)$ & 0.031 \\
\hline
\end{tabular}

Note: *Adjusting for age, gender, stage, radical treatment, multiple lesions, and tumor location.

\section{Discussion}

This was the first study to systematically explore the impact of multiple lines of active treatment in relapsed and metastasis ESCC patients. Two and more lines of aggressive therapy were associated with better prognosis $(\mathrm{cHR}(95 \% \quad \mathrm{CI})=0.21 \quad(0.06-0.73), \quad \mathrm{p}=0.014)$, compared with one line of active therapy only. After adjusting for covariables, including age, gender, stage, previous radical treatment, and lesion numbers and location, multiple active therapy lines were still associated with longer survival $($ aHR $(95 \% \mathrm{CI})=0.19(0.04-0.86), \mathrm{p}=0.031)$.

For metastasis and recurrent ESCC patients, the major purpose of current treatment is palliative, with the aim of alleviating the condition, prolonging survival, and improving quality of life. Although a few patients are cured by palliative 


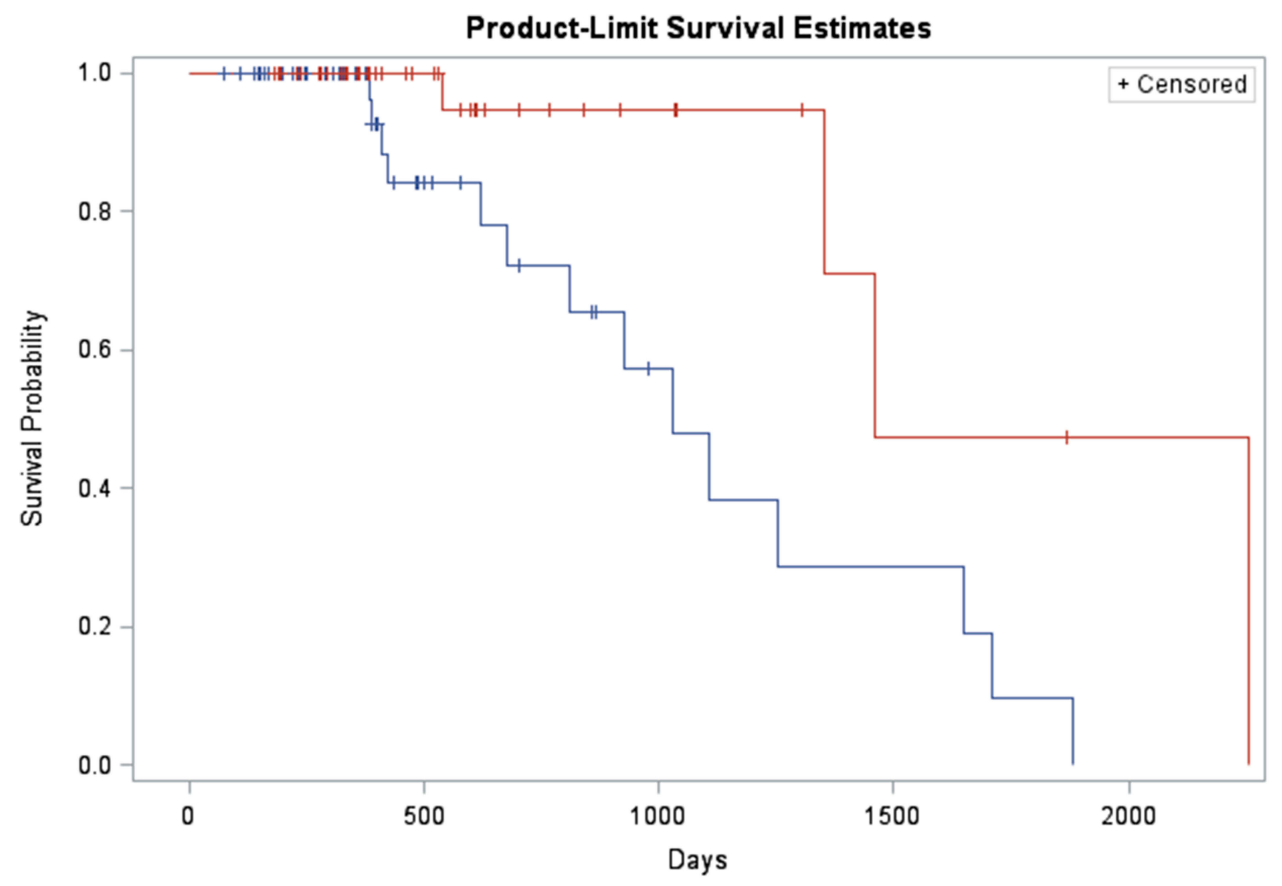

Figure 2 Survival plot of the association between active therapy line counts and overall survival in recurrent and metastasis ESCC patients. Notes: Blue line: only one line of active treatment. Red line: two and more lines of active treatment.

care, the majority respond initially but eventually progress, with a number of patients progressing multiple times. Notably, some of these patients are still able to receive the second- and later-line active treatment despite progressing again. Nevertheless, most previous studies have only focused on first-line chemotherapy regimens or on targeted and immune therapies, such as irinotecan and $\mathrm{S}-1,{ }^{16}$ icotinib, ${ }^{17}$ and immune checkpoint inhibitors. ${ }^{18}$ Even for chemotherapy, there is a lack of large-sample, randomized, double-blinded clinical trials to explore the effects of second- and later-line regimens, ${ }^{16,19}$ let alone other therapy modalities.

With regard to multiple lines of active treatment, there have been several case reports of successful multidisciplinary

Table 3 Adverse Events Indifferent Active Treatment Line Groups

\begin{tabular}{|c|c|c|c|}
\hline & \multicolumn{2}{|c|}{$\begin{array}{l}\text { Grade 3-4 Events/All } \\
\text { Events }\end{array}$} & \multirow[t]{2}{*}{ p-value } \\
\hline & $\begin{array}{l}\text { One } \\
\text { Line }\end{array}$ & $\begin{array}{l}\text { Two and More } \\
\text { Lines }\end{array}$ & \\
\hline $\begin{array}{l}\text { Myelosuppression } \\
\text { (neutrophil) }\end{array}$ & $24 / 42$ & $13 / 33$ & 0.127 \\
\hline Hepatotoxicity & $0 / 18$ & $4 / 15$ & 0.033 \\
\hline Renal toxicity & $0 / 0$ & $0 / 2$ & NA \\
\hline Nerve toxicity & $0 / 13$ & $0 / 8$ & NA \\
\hline
\end{tabular}

treatment in recurrent and metastasis esophageal cancer patients, with therapy modalities including but not limited to salvage surgery, chemoradiotherapy, radiofrequency ablation, and hepatic arterial infusions. ${ }^{10-13}$ Some population studies on second- and third-line chemotherapy have reported prolonged PFS, higher objective response rate, or higher 6-month survival rate; nonetheless, the OS has been disappointing. ${ }^{14,16}$ Thus, the evidence for the benefits of salvage treatment and therapy modality options is inconclusive. The relatively high morbidity and mortality rates following salvage treatment, especially metastasectomy, add to the controversy. ${ }^{20}$

In our retrospective cohort study, no patients received salvage surgery. As the number of progressions increased, more patients received systematic treatment only. No increase in severe side effects was observed in patients receiving multiple lines of active treatment, except grade 3-4 hepatic toxicity, and no treatment-related deaths were reported in our research. However, we did find that receiving more than one line of active treatment prolonged patients' survival., We propose that multiple lines of active treatment are beneficial. Notably, 15 out of 138 patients (10.9\%) receiving palliative management achieved longterm survival and even cure. We did not find unique clinicopathological features in these 15 patients, although survivors were more likely to have received both systematic and local treatment $(60 \%)$ than other patients $(34 \%)$. 
As an explorative study, the limitations of our research included the small sample size, the retrospective nature of the study, and movement of patients among hospitals. This often caused loss of crucial data, such as tumor metastasis patterns, organ involvement, and oligometastasis, which were thought to be associated with treatment regimens and prognosis. ${ }^{21,22}$ Performance status was evaluated in outpatient settings for a number of patients, and the records may have contained inaccuracies and mistakes; thus, the survival advantage may have been inflated by patients in better condition receiving more lines of active treatment. The study was also subject to various biases, including confounding bias, measurement bias, and selection bias, which could have either exaggerated or weakened the associations, thereby affecting the reliability of our conclusions. Finally, due to the heterogeneity of each active treatment line, there was lack of standardized therapeutic strategy. For example, 15 patients cured by first-line treatment emphasizing the importance of comprehensive application of both systematic and local treatment; however, we observed that with disease progressed several times, many patients received chemotherapy only, and local treatment was less common; consequently, the optimal treatment strategy still needs to be established.

\section{Conclusions}

Based on these findings, we believed that in cases of recurrent and metastasis ESCC, if further progression occurred after first-line treatment, two and even more active lines of treatment were appropriate and would improve prognosis. However, our study suffered from several inherent defects, and prospective, large-sample trials are warranted to verify our findings and further establish guidelines for multipleline therapy. Furthermore, exploring the characteristics of patients cured by palliative management - not only clinicopathological attributes such as recurrence and metastasis patterns, but also genetic properties - would help drive the application of precision medicine in the future.

\section{Funding}

The study was funded by the National Natural Science Foundation of China (81602924).

\section{Disclosure}

The authors declare that they have no conflicts of interest relating to this work.

\section{References}

1. Feng RM, Zong YN, Cao SM, Xu RH. Current cancer situation in China: good or bad news from the 2018 global cancer statistics? Cancer Commun (Lond). 2019;39(1):22. doi:10.1186/s40880-0190368-6

2. Chen W, Zheng R, Baade PD, et al. Cancer statistics in China, 2015. CA Cancer J Clin. 2016;66(2):115-132. doi:10.3322/caac.21338

3. Abnet CC, Arnold M, Wei W-Q. Epidemiology of esophageal squamous cell carcinoma. Gastroenterology. 2018;154(2):360-373. doi:10.1053/j.gastro.2017.08.023

4. Miyata H, Yamasaki M, Kurokawa $Y$, et al. Survival factors in patients with recurrence after curative resection of esophageal squamous cell carcinomas. Ann Surg Oncol. 2011;18(12):3353-3361. doi:10.1245/s10434-011-1747-7

5. Rustgi AK, El-Serag HB, Ingelfinger JR. Esophageal carcinoma. $N$ Engl J Med. 2014;371(26):2499-2509. doi:10.1056/NEJMra13 14530

6. Enzinger PC, Ilson DH, Kelsen DP. Chemotherapy in esophageal cancer. Semin Oncol. 1999;26(5 Suppl 15):12-20.

7. Arzu O. Ten-year survival of esophageal cancer after an en-bloc esophagectomy. J Surg Oncol. 2012;3:105.

8. Huang J, Zhou Y, Zhang H, et al. A Phase II study of biweekly paclitaxel and cisplatin chemotherapy for recurrent or metastatic esophageal squamous cell carcinoma: ERCC1 expression predicts response to chemotherapy. Med Oncol. 2013;30(1):343. doi:10. 1007/s12032-012-0343-4

9. Thallinger CM, Raderer M, Hejna M. Esophageal cancer: a critical evaluation of systemic second-line therapy. J Clin Oncol. 2011;29 (35):4709-4714. doi:10.1200/JCO.2011.36.7599

10. Iitaka D, Shiozaki A, Fujiwara H, et al. Case involving long-term survival after esophageal cancer with liver and lung metastases treated by multidisciplinary therapy: report of a case. Surg Today. 2013;43(5):556-561. doi:10.1007/s00595-012-0240-7

11. Suzuki T, Izumi Y, Miura A, Kato T, Kawada K. Successful management of the recurrent esophageal cancer following esophagectomy at a different time with combined local treatment of chemoradiotherapy and hepatic arterial infusion chemotherapy. Gan to Kagaku Ryoho. 2008;35(10):1737-1739.

12. Chen F, Sato K, Sakai H, et al. Pulmonary resection for metastasis from esophageal carcinoma. Interact Cardiovasc Thorac Surg. 2008;7(5):809-812. doi:10.1510/icvts.2008.180778

13. Hirose K, Saeki H, Nakashima Y, et al. Successful multidisciplinary treatment including repeated metastasectomy for recurrent squamous cell esophageal carcinoma: a case report. Surg Case Rep. 2019;5 (1):72. doi:10.1186/s40792-019-0634-5

14. Enzinger PC, Mayer RJ. Esophageal cancer. $N$ Engl J Med. 2003;349 (23):2241-2252. doi:10.1056/NEJMra035010

15. Rice TW, Blackstone EH, Rusch VW. 7th edition of the AJCC cancer staging manual: esophagus and esophagogastric junction. Ann Surg Oncol. 2010;17(7):1721-1724. doi:10.1245/s10434-010-1024-1

16. Huang J, Xu B, Liu Y, et al. Irinotecan plus S-1 versus S-1 in patients with previously treated recurrent or metastatic esophageal cancer (ESWN 01): a prospective randomized, multicenter, open-labeled Phase 3 trial. Cancer Commun (Lond). 2019;39(1):16. doi:10.1186/ s40880-019-0359-7

17. Huang J, Fan Q, Lu P, et al. Icotinib in patients with pretreated advanced esophageal squamous cell carcinoma with EGFR overexpression or EGFR gene amplification: a single-arm, multicenter phase 2 study. J Thorac Oncol. 2016;11(6):910-917. doi:10.1016/j. jtho.2016.02.020

18. Kudo T, Hamamoto Y, Kato K, et al. Nivolumab treatment for oesophageal squamous-cell carcinoma: an open-label, multicentre, phase 2 trial. Lancet Oncol. 2017;18(5):631-639. doi:10.1016/ S1470-2045(17)30181-X 
19. Park BB, Im YH, Hwang IG, et al. Salvage chemotherapy with mitomycin $\mathrm{C}$, ifosfamide, and cisplatin (MIC) for previously treated metastatic or recurrent esophageal squamous cell carcinoma. Invest New Drugs. 2008;26(4):387-392. doi:10.1007/s10637-008-9126-3

20. Cohen C, Tessier W, Gronnier C, et al. Salvage surgery for esophageal cancer: how to improve outcomes? Ann Surg Oncol. 2018;25 (5):1277-1286. doi:10.1245/s10434-018-6365-1
21. Ghaly G, Harrison S, Kamel MK, et al. Predictors of survival after treatment of oligometastases after esophagectomy. Ann Thorac Surg. 2018;105(2):357-362. doi:10.1016/j.athoracsur.2017.10.039

22. Kato F, Monma S, Koyanagi K, et al. Long-term outcome after resection for recurrent oesophageal cancer. J Thorac Dis. 2018;10 (5):2691-2699. doi:10.21037/jtd.2018.05.17

\section{Publish your work in this journal}

Cancer Management and Research is an international, peer-reviewed open access journal focusing on cancer research and the optimal use of preventative and integrated treatment interventions to achieve improved outcomes, enhanced survival and quality of life for the cancer patient.
The manuscript management system is completely online and includes a very quick and fair peer-review system, which is all easy to use. Visit http://www.dovepress.com/testimonials.php to read real quotes from published authors. 